\title{
Diagnosis and Treatment of Mucormycosis in Patients with Hematological Malignancies [Translated Article]
}

\author{
Yuki Asano-Mori \\ Department of Hematology, Toranomon Hospital
}

\begin{abstract}
The risk of invasive fungal infections (IFIs) is extremely high in patients with hematological malignancies due to the prolonged and profound neutropenia and immunosuppression after chemotherapy and hematopoietic stem cell transplantation. There has been increasing interest in mucormycosis despite its relatively uncommon occurrence, because occasional breakthrough infections have been observed under anti-Aspergillus prophylaxis. The aggressive nature of mucormycosis easily leads to high mortality because of delays in diagnosis and incorrect treatment decisions, which are due in part to lack of adjunctive diagnostic tools and having similar clinical and radiological features with invasive aspergillosis. The only currently available antifungals against Mucorales in Japan are amphotericin B formulations. Thus, comprehensive therapeutic strategies, including surgery, should be considered to achieve a successful outcome.
\end{abstract}

Key words : hematological malignancies, invasive fungal infections, liposomal-amphotericin B, mucormycosis

\section{Introduction}

Invasive fungal infections (IFIs) are important complications affecting the prognosis of hematological diseases. Remarkable advances in the treatment of hematopoietic stem cell transplantation (HSCT) have expanded the application to the elderly or patients without an HLA (human leukocyte antigen) matched donor, resulting in the increased risks of IFIs. Although advanced diagnostic techniques including high-resolution CT scans and non-culture based diagnosis, and the development of newer drugs with a broad antifungal spectrum and low toxicity, have improved the prevention and treatment of invasive candidiasis (IC) and invasive aspergillosis (IA), other forms of fungal infection are emerging. One of these is mucormycosis. The difficulty in distinguishing between mucormycosis and IA based on their clinical course or imaging results is a matter, because voriconazole (VRCZ: the first drug of choice for $(A)$ is ineffective against mucormycosis. Although guidelines specific to mucormy- cosis have been published in recent years ${ }^{1,2)}$, we still lack adjunctive diagnosis techniques for mucormycosis and effective antifungal agents are also limited. As a result, the prognosis of mucormycosis is even very poor now. In this paper, we review the current status of mucormycosis in immunocompromised patients with hematological malignancies, and consider how these problems can be overcome and the future prospects for its management.

\section{Epidemiology of mucormycosis}

Mucormycosis, previously known as zygomycosis, was renamed following the elimination of the zygomycota phylum based on a phylogenetic classification using molecular biological techniques. It is now considered to belong to the pathogenic fungi that are mainly in the Mucorales order of the newly classified mucoromycotina subphylum. Although mucormycosis also occurs in immunocompetent patients ${ }^{3)}$ and its reported frequency differs between institutions, the frequency is significantly higher and the prognosis is 
extremely poor in immunocompromised patients.

Most cases of mucormycosis are currently observed in hematological patients, in spite of the common occurrence in diabetes patients previously ${ }^{4)}$. A prospective multicenter study conducted for HSCT patients between 2001 and 2006 by the Transplant Associated Infection Surveillance Network (TRANSNET) reported that the frequency of mucormycosis came in third place $(8 \%)$ after IA $(43 \%)$ and IC $(28 \%)$, and the cumulative incidence at one year after transplantation was $0.29 \%{ }^{5}$. In another prospective study for HSCT patients between 2008 and 2010 by the Gruppo Italiano Trapianto Midollo Osseo (GITMO), an incidence of mucormycosis was $3.7 \%$, following IA $(81.1 \%)$ and IC $(11 \%)^{6}$. A prospective multicenter study for patients with IFIs by the PATH (Prospective Antifungal Therapy) Alliance identified mucormycosis in 18 out of overall $250 \mathrm{IFI}$ cases $(7.2 \%)^{7}$. Although the incidence of mucormycosis is relative rare even in high-risk hematological patients, the onset might be underestimated due to the difficulty of ante-mortem diagnosis, and therefore observing future trends is very important

The fungi responsible for mucormycosis in humans include Rhizopus spp., Mucor spp., Rhizomucor spp., Cunninghamella spp. and Lichtheimia spp. The identification of fungal species is extremely difficult, because mucormycosis is mostly diagnosed by pathological examination and predominantly confirmed by autopsy. The TRANSNET study reported Rhizopus spp. as the most common (55 cases $=52.4 \%$ ), followed by Mucor spp. (15.6\%) and Rhizomucor spp. (9.1\%) (Table 1) $)^{8)}$, and the PATH alliance study also reported Rhizopus spp. as the most common (52. 1\%), followed by Mucor spp. (23.1\%). In contrast with the results of these two studies in North America, the Lichtheimia spp. is relatively common in Europe $^{10,111}$. Although there is no large-scale clinical research of mucormycosis in Japan, the distribution of pathogenic fungi seems to be slightly different from those in Europe and the United States ${ }^{12)}$.

Mucormycosis includes five disease types: pulmonary, rhino-orbito-cerebral, gastrointestinal, cutaneous and disseminated ${ }^{13)}$. The fungus mainly attaches to the nasal passages or respiratory tract, penetrates through the airways and often forms lesions in the lungs or nasal cavity (Fig. 1). It may sometimes enter via the gastrointestinal tract, or occasionally propagates to multiple organs via the blood stream if the host immunity decreases. The sites of infection vary with underlying disease, the main of which are lungs followed by rhinocranial region in hematological patients ${ }^{4,9,10)}$.

\section{Risk factors of mucormycosis}

As with IA, risk factors of mucormycosis include prolonged severe neutropenia, high-risk transplantations, graft-versus-host disease (GVHD) and its treatments, prolonged use of high-dose steroids, cytomegalovirus infections and previous IFIs. Iron overload, administration of deferoxamine, persistent hyperglycemia and poorly controlled diabetes, and the use of VRCZ are relatively characteristic of mucormycosis (Fig. $1)^{14-16)}$. When the level of free iron, normally controlled by transferrin, is increased, excess iron is used for fungal growth ${ }^{17}$. Although deferoxamine is the iron chelating agent, it binds with iron to form siderophores which act as iron donors to fungi and promote fungal growth. Diabetes and hyperglycemia are independent risk factors for mucormycosis in patients with or without hematological diseases ${ }^{3}$. When high-dose steroids are administered, the migratory and fungicidal abilities of phagocytes are diminished, and their ability to prevent infection is suppressed. VRCZ has been regarded as a risk factor, and breakthrough infections while on VRCZ have been reported ${ }^{18,19)}$. However, careful consideration should be given to the fact that patients receiving VRCZ prophylaxis are generally at high risk for IFIs, and extremely like to have other factors related to an increased risk of IFIs including prolonged neutropenia and the use of steroids. The assessment of detection frequency at each medical facility is important, because the onset of mucormycosis tends to accumulate at same facilities.

\section{Diagnosis of mucormycosis}

The first step towards a definitive diagnosis of mucormycosis is "doubt it". Mucormycosis should be always kept in mind in the differential diagnosis of IFls, because its clinical symptoms and imaging findings are similar to those of IA, and there are few adjunctive diagnosis methods besides pathological examination. 






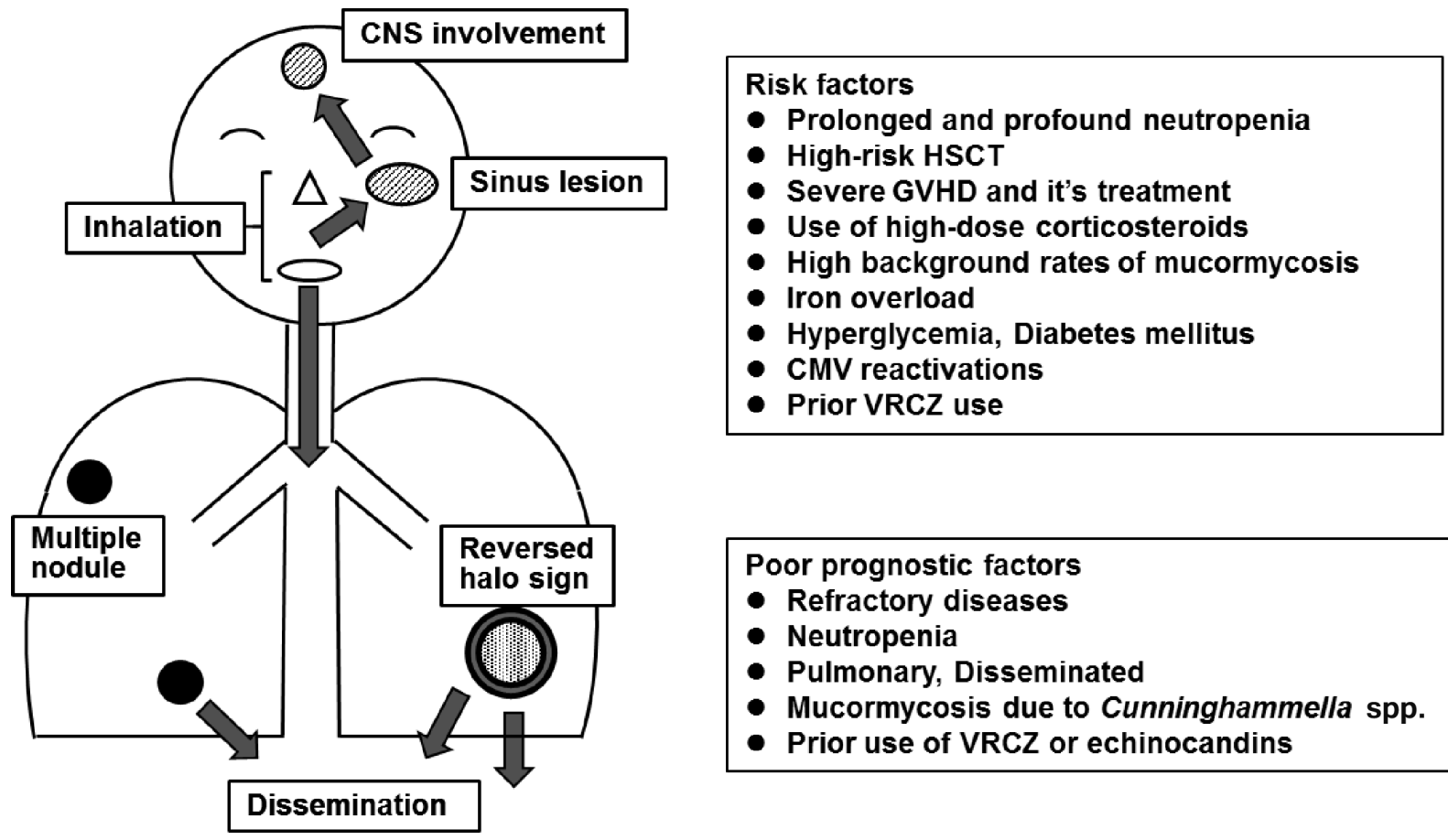

Fig. 1 Clinical manifestations, risk and prognostic factors of mucormycosis. CNS: central nervous system, HSCT: hematopoietic stem cell transplantation, GVHD: graft-versus-host disease, VRCZ: voriconazole

\section{Diagnostic imaging}

Although distinguishing pulmonary mucormycosis from invasive pulmonary aspergillosis (IPA) is difficult due to the similar clinical features and CT findings, there are a few signs indicative of mucormycosis, such as the presence of multiple nodules at 10 or more sites, pleural effusion, reversed halo sign (RHS), and cellulitis of the thoracic wall adjacent to pulmonary infarctions ${ }^{15,20,21}$. RHS is defined as central ground-glass opacity surrounded by a complete or nearly complete ring of dense consolidation, as demonstrated on high-resolution CT scan. The central ground-glass opacity corresponds to coagulation necrosis which presents as reticular pattern, because the fungus spreads along alveolar lumen without disrupting the blood vessels and air content is remained in alveolar space. The ringshaped consolidation corresponds to granulomatous tissue, because severe bleeding or inflammation are caused by neutrophils and macrophages attack to the outwardly expanding fungi during immune restoration ${ }^{22)}$. RHS must be useful in the early diagnosis, because it is observed more often in mucormycosis than in IPA and appears in the early stage ${ }^{20)}$.

A sinus CT scan is also recommended in patients with pulmonary lesions suspicious of mucormycosis, because sinusitis is presented as a complication more frequently than IPA ${ }^{21)}$. Mucosal necrosis of the palate and nasal cavity, together with orbital cellulitis, cavernous sinus thrombosis or cerebral abscess is observed in some cases. When sinus lesions are found, a brain CT scan or MRI is preferably performed to screen central nervous system (CNS) lesions. Intensification of treatment is required for CNS mucormycosis ${ }^{11}$.

2. Culturing and histopathological diagnosis

Unlike in the case of IA, there are no specific antigen tests for mucormycosis, and the $\beta-D$ glucan test results are negative due to its small amount of production. Although a positive $\beta$-Dglucan test result can not rule out mucormycosis, a negative result of Aspergillus galactomannan antigen suggests the possibility of mucormycosis".

Mucorales is angioinvasive, but hardly ever yields a positive blood culture test result. Although it is important to repeatedly collect clinical specimens such as sputum, bronchoalveolar lavage fluid, pleural fluid, nasal secretions and sinus aspirate, the positive culture rate is not high and therefore diagnosis is principally made by histopathological examination from biopsy samples. Even if the fungus is pathologically confirmed the culture can be still negative in some cases. 
Although biopsies are often difficult due to the increased susceptibility of infection or bleeding in hematological patients, the usefulness of CTguided needle biopsy has been reported ${ }^{23}$. Histopathological characteristics have the potential to distinguish Mucorales from Aspegillus species. Mucorales produce broad, aseptate hyphae branching irregularly at obtuse angles ${ }^{24}$, whereas Aspergillus species are thinner, septate, with regular branching at acute angles. Along with the difficulty in detecting hyphae on biopsy, coexistence with other fungal infections make it difficult to diagnose mucormycosis in high-risk hematological patients. However, the greatest efforts possible should be made to identify the fungal species, because the differences in treatment response and prognosis depending on the fungal strains have been suggested ${ }^{25}$. Although advances have been made in molecular identification of fungal species including identification by DNA sequencing, identification by PCR methods specific to the Mucorales, and identification by in situ hybridization using pathology sections ${ }^{24}$, none of them have been standardized and therefore specialist consultation is necessary.

\section{Prevention of mucormycosis}

Antifungal prophylaxis is primarily targeted at IC and IA, and prophylaxis against mucormycosis is not generally performed. However, species of Mucorales widely distributed in the world, despite being not as common as Aspergillus spp. Careful environment management is therefore essential, based on several reports of outbreaks during medical implements and rebuilding works ${ }^{26}$. Posaconazole is expected to be effective as prophylaxis of mucormycosis, which is not currently available in Japan. Two prospective studies comparing posaconazole with fluconazole (FLCZ) or itraconazole (ITCZ) for prophylaxis against IFIs in allogeneic HSCT recipients with severe GVHD and in prolonged neutropenic patients with acute myelogenous leukemia or myelodysplastic syndrome reported one case of mucormycosis in the control (FLCZ or ITCZ) group, compared with none in the posaconazole group ${ }^{27,28)}$. However, there has even been a report of a breakthrough infection while on posaconazole ${ }^{29}$, and therefore the efficacy of prophylactic posaconazole is difficult to discuss solely on the basis of these results.

\section{Treatment of mucormycosis}

There are three approaches that can be considered for the treatment of mucormycosis: (1) elimination of risk factors, (2) treatment with antifungal drugs, and (3) surgical excision.

1. Elimination of risk factors

Neutropenia and immunosuppression are key risk factors in hematological patients, which can be reduced by protected environment (high efficiency particulate air (HEPA) filters), the use of granulocyte-colony stimulating factor (G$(\mathrm{CSF})^{2)}$, and tapering of immunosuppressants and steroids if possible. Attention to a breakthrough infection under VRCZ administration should be payed, because VRCZ reportedly has the potential to enhance the pathogenicity of Mucorales ${ }^{30}$. Management of transfusion-induced iron overload (by administering deferasirox) and hyperglycemia is also important.

2. Treatment with antifungal drugs

Immediate initiation of high-dose amphotericin B formulations is essential in cases where mucormycosis cannot be ruled out based on the clinical course and laboratory findings, because mucormycosis is difficult to distinguish from IA and to diagnose ante-mortem, and its causative agents are resistant to VRCZ. Amphotericin B and its liposomal formulation called liposomalamphotericin B (L-AMB) are currently available in Japan, the latter of which is recommended due to the lower renal toxicity and better CNS penetration.

The treatment with L-AMB at $5 \mathrm{mg} / \mathrm{kg}$ or more is recommended for mucormycosis, because of the poorer response compared with $\mid \mathrm{A}^{1,2,15)}$. The use of high-dose L-AMB should not be hesitated even in patients receiving nephrotoxic drugs, with adequate replacement fluids and strict electrolyte management. We have experienced a fatal case of pulmonary mucormycosis that was hardly distinguishable from IPA and firstly treated with 3 $\mathrm{mg} / \mathrm{kg}$ of $\mathrm{L}-\mathrm{AMB}^{22}$. Although currently available dose of L-AMB is $5 \mathrm{mg} / \mathrm{kg}$ in Japan and the superiority of initial treatment with L-AMB at 10 $\mathrm{mg} / \mathrm{kg}$ was not shown in a recent prospective preliminary study ${ }^{31}$, an increased dose of 7.5-10 $\mathrm{mg} / \mathrm{kg}$ is recommended as an expert opinion ${ }^{14}$. The use of L-AMB at $10 \mathrm{mg} / \mathrm{kg}$ is recommended for the treatment of CNS mucormycosis by the European guidelines ${ }^{1,2)}$. The dosage of L-AMB 
should be therefore individualized for each patient, by reference to patient's background, organ functions including renal function, and the progress of mucormycosis.

The initiation timing of L-AMB is also important ${ }^{9}{ }^{92}$. Chamilos et al. reported a 2-fold increase in mortality rate at 12 weeks after diagnosis in the delayed treatment group (initiating treatment $\geq 6$ days after diagnosis) compared to the early treatment group $(82.9 \% \text { versus. } 48.6 \%)^{32}$. A significant association between the timing of initiation and the prognosis has been also reported by Kontoyiannis et al., based on the result that the treatment initiation within 3 days led to better survival ${ }^{9}$. Prompt initiation of treatment is highly desirable in cases where mucormycosis cannot be ruled out, because even a slight delay in treatment potentially result in a lethal outcome.

Although a treatment duration with L-AMB has been controversial, it is recommended to continue until the image findings are completely improved and all risk factors have been eliminated (e. g., until immunosuppressants are no longer being administered), and at least for a continuous period of 6-8 weeks. For L-AMB intolerant patients, or as maintenance therapy after an initial treatment of $\mathrm{L}-\mathrm{AMB}$, the administration of posaconazole is recommended in Europe and the United States ${ }^{33,34)}$, and the effectiveness of isavuconazole against mucormycosis has been recently reported $^{35)}$. However, neither of these has yet been approved in Japan.

For combination therapy, Pagano et al. reported the beneficial effect of posaconazole in addition to $L-A M B$ in hematological patients failing to response to L-AMB monotherapy ${ }^{36}$. Concomitant use of echinocandins with amphtericin B formulations has also been reported to show improved efficacy, because synergetic effect was obtained in spite of little in vivo activity of echinocandins against Mucorales ${ }^{377}$. However, the effectiveness of combination therapy with echinocandins and amphotericin B formulations is not confirmed in other studies ${ }^{38,39}$, and therefore it is still controversial. At least in the cases where the first-line therapy is ineffective, the combination therapy is worthy of consideration.

Deferasirox is a new iron chelator which does not act as a siderophore, and suppresses the proliferation of Mucorales by depriving it of iron. However, in a recent prospective randomized controlled trial comparing the treatment with L-
AMB plus deferasirox with the treatment with L$A M B$ plus placebo, the effectiveness of concomitant deferasirox with L-AMB was not demonstrated, and the mortality rate was higher in the deferasirox group ${ }^{40}$. A definitive conclusion has not yet been reached about concomitant use of deferasirox and L-AMB, because the deferosirox group has included more patients with hematological malignancy or neutropenia. Concomitant deferasirox therapy is discouraged in the European guidelines on mucormycosis ${ }^{1,2)}$.

\section{Surgical excision}

Infected tissues should be surgically removed if feasible. Especially in cases with rhino-orbitocerebral mucormycosis, surgical debridement is recommended for local control and elimination of necrotic tissue which are poorly penetrated by antifungal agents ${ }^{1,2,41)}$

On the other hand, pulmonary mucormycosis, mainly seen in hematological patients, are difficult to excise in most cases due to increased susceptibility of infection and bleeding, and therefore surgical resection is made available as an option in less-severely ill patients with unifocal lesion ${ }^{42,43)}$. Surgical resection for pulmonary mucormycosis has been reported to lead to a better prognosis ${ }^{10,11}$, and we have also experienced successful cases by a combination of surgical excision and antifungal therapy with high-dose L-AMB despite profound immunosuppression and severe neutropenia in the early period after allogeneic $\mathrm{HSCT}^{44)}$. Surgical intervention is crucial and should be considered under antifungal therapy coupled with ongoing treatment for underlying disease and enhancement of immune recovery.

\section{Prognosis of mucormycosis}

Mortality depends on factors including underlying disease, site of infection, fungal species, and therapeutic regimen. The prognosis is poor in patients with active malignancy or neutropenia ${ }^{45}$. The mortality rate of pulmonary mucormycosis (the most common type) in hematological patients reaches approximately $75 \%$, and can be even higher in highly immunosuppressed patients with disseminated diseases ${ }^{4,45}$. The detection of Cunninghamella spp. is an independent factor for a poor prognosis ${ }^{4,25}$. Although a poor prognosis of breakthrough infections while on caspofungin and/or VRCZ has been also reported ${ }^{9}{ }^{10)}$, several questions remain unanswered, such as whether 
these antifungal agents cause an increase in pathogenicity ${ }^{30)}$, or whether the frequent use of these agents leads to delay of diagnosis in highrisk patients .

\section{Conclusion}

Although consensus recommendations have been gradually established and practical guidelines have recently published for the diagnosis and treatment of mucormycosis, there is still insufficient evidence to yield therapeutic success compared with other fungal infections. Despite greater risks for mucormycosis in hematological patients, ante-mortem diagnosis is not feasible due to increased bleeding risk and susceptibility of infections. There are no antifungal agents approved for prophylaxis and only a limited number of choice for treatment. The timing for surgical intervention, the proper doses of L-AMB depending on fungal species, and the pros and cons of combination therapy still remain as unsolved issues.

The most important things are to consider mucormycosis constantly in the differential diagnosis of IFIs, and to start high-dose L-AMB immediately when mucormycosis cannot be rules out based on the clinical course and findings. A major step towards overcoming mucormycosis would be the comprehensive diagnosis and treatment in close cooperation with respiratory physicians, thoracic surgeons, infectious disease specialists and pathologists, for definite diagnosis, identification of fungal strains, and prompt surgical intervention.

\section{Conflict of interest}

None.

\section{References}

1) Skiada $A$, Lanternier $F$, Groll $A H$, Pagano $L$, Zimmerli S, Herbrecht R, Lortholary O, Petrikkos GL; European Conference on Infections in Leukemia: Diagnosis and treatment of mucormycosis in patients with hematological malignancies: guidelines from the 3rd European Conference on Infections in Leukemia (ECIL 3). Haematologica 98: 492-504, 2013.

2) Cornely OA, Arikan-Akdagli S, Dannaoui E, et al; European Society of Clinical Microbiology and Infectious Diseases Fungal Infection Study Group;
European Confederation of Medical Mycology: ESCMID and ECMM joint clinical guidelines for the diagnosis and management of mucormycosis 2013. Clin Microbiol Infect 20 Suppl 3: 5-26, 2014.

3) Chakrabarti A, Das A, Mandal J, Shivaprakash MR, George VK, Tarai B, Rao P, Panda N, Verma SC, Sakhuja V: The rising trend of invasive zygomycosis in patients with uncontrolled diabetes mellitus. Med Mycol 44: 335-342, 2006.

4) Roden MM, Zaoutis TE, Buchanan WL, Knudsen TA, Sarkisova TA, Schaufele RL, Sein M, Sein T, Chiou CC, Chu JH, Kontoyiannis DP, Walsh TJ: Epidemiology and outcome of zygomycosis: a review of 929 reported cases. Clin Infect Dis 41: 634-653, 2005.

5) Kontoyiannis DP, Marr KA, Park BJ, et al: Prospective surveillance for invasive fungal infections in hematopoietic stem cell transplant recipients, 2001-2006: overview of the TransplantAssociated Infection Surveillance Network (TRANSNET) Database. Clin Infect Dis 50: 10911100, 2010.

6) Girmenia C, Raiola AM, Piciocchi A, et al: Incidence and outcome of invasive fungal diseases after allogeneic stem cell transplantation: a prospective study of the Gruppo Italiano Trapianto Midollo Osseo (GITMO). Biol Blood Marrow Transplant 20: 872-880, 2014.

7) Neofytos D, Horn D, Anaissie E, Steinbach W, Olyaei A, Fishman J, Pfaller M, Chang C, Webster $\mathrm{K}$, Marr K: Epidemiology and outcome of invasive fungal infection in adult hematopoietic stem cell transplant recipients: analysis of Multicenter Prospective Antifungal Therapy (PATH) Alliance registry. Clin Infect Dis 48: 265-273, 2009.

8) Park BJ, Pappas PG, Wannemuehler KA, et al: Invasive non-Aspergillus mold infections in transplant recipients, United States, 2001-2006. Emerg Infect Dis 17: 1855-1864, 2011.

9) Kontoyiannis DP, Azie N, Franks B, Horn DL: Prospective antifungal therapy (PATH) alliance ${ }^{\mathrm{B}}$ : focus on mucormycosis. Mycoses 57: 240-246, 2014.

10) Skiada A, Pagano L, Groll A, et al; European Confederation of Medical Mycology Working Group on Zygomycosis: Zygomycosis in Europe: analysis of 230 cases accrued by the registry of the European Confederation of Medical Mycology (ECMM) Working Group on Zygomycosis between 2005 and 2007. Clin Microbiol Infect 17: 1859-1867, 2011.

11) Lanternier F, Dannaoui E, Morizot G, Elie C, GarciaHermoso D, Huerre M, Bitar D, Dromer F, Lortholary O; French Mycosis Study Group: A global analysis of mucormycosis in France: the RetroZygo Study (2005-2007). Clin Infect Dis 54 Suppl 1: S35-43, 2012.

12) Mori T, Yahata $Y$, Tsukune $Y$ : [Zygomycosis]. Med Mycol J 52: 283-289, 2011.

13) Petrikkos G, Skiada A, Lortholary O, Roilides E, Walsh TJ, Kontoyiannis DP: Epidemiology and 
clinical manifestations of mucormycosis. Clin Infect Dis 54 Suppl 1: S23-34, 2012.

14) Kontoyiannis DP, Lewis RE: How I treat mucormycosis. Blood 118: 1216-1224, 2011.

15) Guidelines for Management of Deep-seated Mycosis 2014, pp. 120, Kyowa Kikaku, Tokyo, 2014. [Contents in Japanese]

16) Kontoyiannis DP, Lionakis MS, Lewis RE, Chamilos G, Healy M, Perego C, Safdar A, Kantarjian H, Champlin R, Walsh TJ, Raad II: Zygomycosis in a tertiary-care cancer center in the era of Aspergillus-active antifungal therapy: a casecontrol observational study of 27 recent cases. J Infect Dis 191: 1350-1360, 2005.

17) Ibrahim AS, Kontoyiannis DP: Update on mucormycosis pathogenesis. Curr Opin Infect Dis 26: 508-515, 2013.

18) Trifilio SM, Bennett CL, Yarnold PR, McKoy JM, Parada J, Mehta J, Chamilos G, Palella F, Kennedy L, Mullane K, Tallman MS, Evens A, Scheetz $\mathrm{MH}$, Blum W, Kontoyiannis DP: Breakthrough zygomycosis after voriconazole administration among patients with hematologic malignancies who receive hematopoietic stem-cell transplants or intensive chemotherapy. Bone Marrow Transplant 39: 425-429, 2007.

19) Siwek GT, Dodgson KJ, de Magalhaes-Silverman M, Bartelt LA, Kilborn SB, Hoth PL, Diekema DJ, Pfaller MA: Invasive zygomycosis in hematopoietic stem cell transplant recipients receiving voriconazole prophylaxis. Clin Infect Dis 39: $584-587$, 2004.

20) Legouge C, Caillot D, Chrétien ML, Lafon I, Ferrant E, Audia S, Pagès PB, Roques M, Estivalet L, Martin L, Maitre T, Bastie JN, Dalle F: The reversed halo sign: pathognomonic pattern of pulmonary mucormycosis in leukemic patients with neutropenia? Clin Infect Dis 58: 672-678, 2014.

21) Chamilos G, Marom EM, Lewis RE, Lionakis MS, Kontoyiannis DP: Predictors of pulmonary zygomycosis versus invasive pulmonary aspergillosis in patients with cancer. Clin Infect Dis 41: 60-66, 2005.

22) Kimura M, Araoka H, Uchida N, Ohno H, Miyazaki Y, Fujii T, Nishida A, Izutsu K, Wake A, Taniguchi S, Yoneyama A: Cunninghamella bertholletiae pneumonia showing a reversed halo sign on chest computed tomography scan following cord blood transplantation. Med Mycol 50: 412-416, 2012.

23) Lass-Flörl C, Resch G, Nachbaur D, Mayr A, Gastl G, Auberger J, Bialek R, Freund MC: The value of computed tomography-guided percutaneous lung biopsy for diagnosis of invasive fungal infection in immunocompromised patients. Clin Infect Dis 45: e101-104, 2007.

24) Walsh TJ, Gamaletsou MN, McGinnis MR, Hayden RT, Kontoyiannis DP: Early clinical and laboratory diagnosis of invasive pulmonary, extrapulmonary, and disseminated mucormycosis (zygomycosis). Clin Infect Dis 54 Suppl 1: S55-60, 2012.
25) Gomes MZ, Lewis RE, Kontoyiannis DP: Mucormycosis caused by unusual mucormycetes, nonRhizopus, -Mucor, and -Lichtheimia species. Clin Microbiol Rev 24: 411-445, 2011.

26) Rammaert B, Lanternier F, Zahar JR, Dannaoui E, Bougnoux ME, Lecuit M, Lortholary O: Healthcareassociated mucormycosis. Clin Infect Dis $54 \mathrm{Suppl}$ 1: S44-54, 2012.

27) Cornely OA, Maertens J, Winston DJ, Perfect J, Ullmann AJ, Walsh TJ, Helfgott D, Holowiecki J, Stockelberg D, Goh YT, Petrini M, Hardalo C, Suresh R, Angulo-Gonzalez D: Posaconazole vs. fluconazole or itraconazole prophylaxis in patients with neutropenia. N Engl J Med 356: 348-359, 2007.

28) Ullmann AJ, Lipton JH, Vesole DH, Chandrasekar P, Langston A, Tarantolo SR, Greinix H, Morais de Azevedo W, Reddy V, Boparai N, Pedicone L, Patino H, Durrant S: Posaconazole or fluconazole for prophylaxis in severe graft-versus-host disease. N Engl J Med 356: 335-347, 2007.

29) Lerolle N, Raffoux E, Socie G, Touratier S, Sauvageon H, Porcher R, Bretagne S, Bergeron A, Azoulay E, Molina JM, Lafaurie M: Breakthrough invasive fungal disease in patients receiving posaconazole primary prophylaxis: a 4-year study. Clin Microbiol Infect 20: O952-959, 2014.

30) Lamaris GA, Ben-Ami R, Lewis RE, Chamilos G, Samonis G, Kontoyiannis DP: Increased virulence of Zygomycetes organisms following exposure to voriconazole: a study involving fly and murine models of zygomycosis. J Infect Dis 199: 1399-1406, 2009.

31) Lanternier F, Poiree S, Elie C, Garcia-Hermoso D, Bakouboula P, Sitbon K, Herbrecht R, Wolff M, Ribaud P, Lortholary O; French Mycosis Study Group: Prospective pilot study of high-dose (10 $\mathrm{mg} / \mathrm{kg} /$ day) liposomal amphotericin B (L-AMB) for the initial treatment of mucormycosis. J Antimicrob Chemother 70: 3116-3123, 2015.

32) Chamilos G, Lewis RE, Kontoyiannis DP: Delaying amphotericin B-based frontline therapy significantly increases mortality among patients with hematologic malignancy who have zygomycosis. Clin Infect Dis 47: 503-509, 2008.

33) van Burik JA, Hare RS, Solomon HF, Corrado ML, Kontoyiannis DP: Posaconazole is effective as salvage therapy in zygomycosis: a retrospective summary of 91 cases. Clin Infect Dis 42: e61-65, 2006.

34) Greenberg RN, Mullane K, van Burik JA, et al: Posaconazole as salvage therapy for zygomycosis. Antimicrob Agents Chemother 50: 126-133, 2006.

35) Marty FM, Ostrosky-Zeichner L, Cornely OA, et al; VITAL and FungiScope Mucormycosis Investigators: Isavuconazole treatment for mucormycosis: a single-arm open-label trial and case-control analysis. Lancet Infect Dis 16: 828-837, 2016.

36) Pagano L, Cornely OA, Busca A, et al: Combined 
antifungal approach for the treatment of invasive mucormycosis in patients with hematologic diseases: a report from the SEIFEM and FUNGISCOPE registries. Haematologica 98: e127-130, 2013.

37) Reed C, Bryant R, Ibrahim AS, Edwards J, Jr., Filler SG, Goldberg R, Spellberg B: Combination polyene-caspofungin treatment of rhino-orbitalcerebral mucormycosis. Clin Infect Dis 47: 364-371, 2008.

38) Abidi MZ, Sohail MR, Cummins N, et al: Stability in the cumulative incidence, severity and mortality of 101 cases of invasive mucormycosis in highrisk patients from 1995 to 2011: a comparison of eras immediately before and after the availability of voriconazole and echinocandin-amphotericin combination therapies. Mycoses 57: 687-698, 2014.

39) Kyvernitakis A, Torres HA, Jiang $Y$, Chamilos G, Lewis RE, Kontoyiannis DP: Initial use of combination treatment does not impact survival of 106 patients with haematologic malignancies and mucormycosis: a propensity score analysis. Clin Microbiol Infect, 2016. doi: 10.1016/j.cmi.2016.03.029. [Epub ahead of print]

40) Spellberg B, Ibrahim AS, Chin-Hong PV, Kontoyiannis DP, Morris MI, Perfect JR, Fredricks D, Brass EP: The Deferasirox-AmBisome Therapy for Mucormycosis (DEFEAT Mucor) study: a randomized, double-blinded, placebo-controlled trial. J Antimicrob Chemother 67: 715-722, 2012.
41) Vironneau P, Kania R, Morizot G, Elie C, GarciaHermoso D, Herman P, Lortholary O, Lanternier F; French Mycosis Study Group: Local control of rhino-orbito-cerebral mucormycosis dramatically impacts survival. Clin Microbiol Infect 20: O336-339, 2014.

42) Chretien ML, Legouge C, Pagès PB, Lafon I, Ferrant E, Plocque A, Favennec C, Estivalet L, Bottolier-Lemallaz E, Dalle F, Bastie JN, Bernard A, Caillot D: Emergency and elective pulmonary surgical resection in haematological patients with invasive fungal infections: a report of 50 cases in a single centre. Clin Microbiol Infect, 2016. doi: 10. 1016/j.cmi.2015.12.029. [Epub ahead of print]

43) Tedder M, Spratt JA, Anstadt MP, Hegde SS, Tedder SD, Lowe JE: Pulmonary mucormycosis: results of medical and surgical therapy. Ann Thorac Surg 57: 1044-1050, 1994.

44) Ota H, Yamamoto $H$, Kimura M, et al: Successful Treatment of Pulmonary Mucormycosis Caused by Cunninghamella bertholletiae with High-Dose Liposomal Amphotericin B (10 mg/kg/day) Followed by a Lobectomy in Cord Blood Transplant Recipients. Mycopathologia 2017. doi: 10. 1007/ s11046-017-0149-1. [Epub ahead of print]

45) Spellberg B, Kontoyiannis DP, Fredricks D, Morris MI, Perfect JR, Chin-Hong PV, Ibrahim AS, Brass EP: Risk factors for mortality in patients with mucormycosis. Med Mycol 50: 611-618, 2012.

This article is based on a study first reported in the Medical Mycology Journal Vol.57 No.4, J155-J162, with full reference. 